THE CLASSIFICATION OF THE STARS ACCORDING TO THEIR TEMPERATURE AND CHEMISTRY.I

\title{
II.
}

THE temperature relationships of the various groups in the classification of stars described in the previous article are further illustrated in a diagram which is reproduced in Fig. 6, from which it will be seen that the stars

$$
\text { Highest temperature. }
$$

9

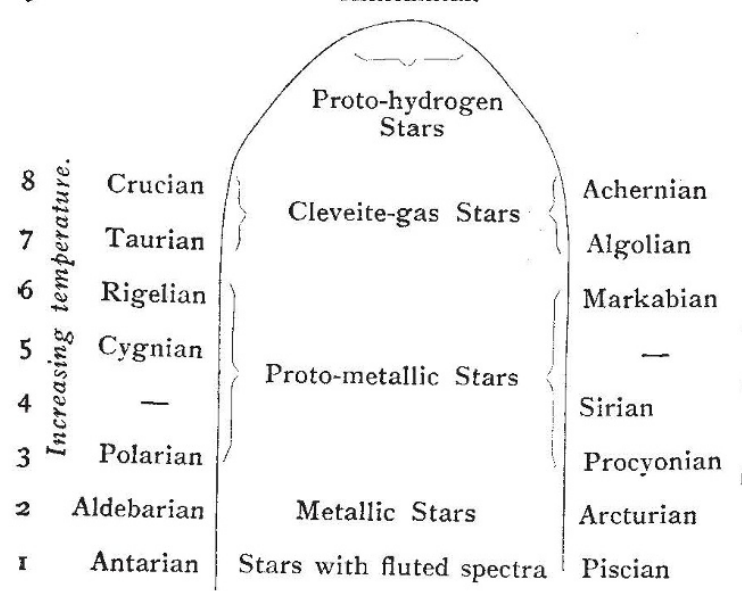

F1G. 6.-Temperature relationship of stellar groups.

are arranged in sixteen groups along a temperature curve having its apex in the middle. Apart from the inferences as to low temperature which may be drawn from the occurrence of flutings, it will be remembered that while the evidence for temperature was primarily based on the strength of the continuous radiation in the violet this detailed arrangement of the stellar groups depended upon the chemical sequence revealed by the successive predominance of metallic, proto-metallic, and "gaseous " lines in the different stars.

This chemical sequence, however, so far as could be judged from the photographs then available, was identical with that derived from the investigation of the violet radiation, thus showing that the apparent chemical differences resulted from changes of temperature. $T \mathrm{~h}$ simplest explanation of the chemical changes is that afforded by the dissociation hypothesis, according to which the step from metallic to protometallic vapours is to be regarded as a breaking up of complex mole cular groupings into simpler ones still retaining characteristics which permit the parent substance to distinguished, while the continuation of the process results in the reduction of all substances to the finer forms of hydrogen and helium. The classification of the stars on a temperature basis is therefore of the utmost importance, not only for the indications which it gives as to the processes of stellar evolution, but also on account of the light which it throws on the dissociation hypothesis and the evolution of the chemical elements. Adopting the foregoing temperature sequence of the various stellar groups, this side of the question has already been fully dealt with by Sir Norman Lockyer in his work on "Inorganic Evolution" (Macmillan and Co., Ltd., rgoo).

$$
1 \text { Continued from p. } 61_{4}
$$

NO. I 826 , vOL. 70$]$
In view of the important issues depending upon the correct determination of relative stellar temperatures, Sir Norman Lockyer has endeavoured to confirm his previous results by another piece of work, of which an account was recently communicated to the Royal Society. ${ }^{1}$ The previous conclusions involving the intensity of the violet radiations depended upon photographs taken with optical appliances composed of glass, which has a marked absorption for these rays, and the relative intensities were judged by noting the limits of the spectra in photographs which were of the same intensity in the region about $\mathrm{H} \gamma$. Although there was no reason to suppose that the general laws of continuous radiation would be modified in still more refrangible parts of the spectrum, it was possible to test the results further by including this region in the discussion. An instrument which was transparent to the ultra-violet radiations was necessary for this research, and the one devised for the purpose was a prismatic camera having a 2-inch $30^{\circ}$ calcite prism mounted in front of a $2 \frac{1}{2}$-inch quartz lens of 18 inches focal length (Fig. 7). The prism is so cut that its first face is perpendicular to the optic axis of the crystal, and it is so arranged that the incident rays are normal to this face. All the rays, therefore, pass through the prism parallel to the optic axis, and there is consequently no double refraction.

By this means it became possible to utilise not only the length of the spectrum in the violet, but the relative brightness of the different parts to a greater extent than before.

To make the matter clear, it may be pointed out that the temperatures of most of the stars are too high to permit of their determination from the actual limits of the continuous radiations towards the violet, as might conceivably be done in the case of a red-hot polser, since these limits lie beyond the wave-length for which our atmosphere is transparent. The principle involved in the method employed, however, is clearly indicated by Sir Norman Lockyer in a quotation from Sir George Stokes, ${ }^{2}$ the substance of which has been fully borne out by more recent work, namely, that

"When a solid body such as a platinum wire, traversed by a voltaic current, is heated to incandescence, we know that as the temperature increases, not only does the radiation of each particular refrangibility absolutely increase, but the proportion of the radiations of the different refrangibilities is changed, the proportion of the higher to the lower increasing with the temperature."

In the case of stars, the radiation is of course modified by the continuous absorption of the stellar gases and vapours; but, so far as we know, the greater absorption is always associated with reduced temperature ${ }^{3}$ and increased density, and regularly diminishes in intensity from the ultraviolet towards the red.

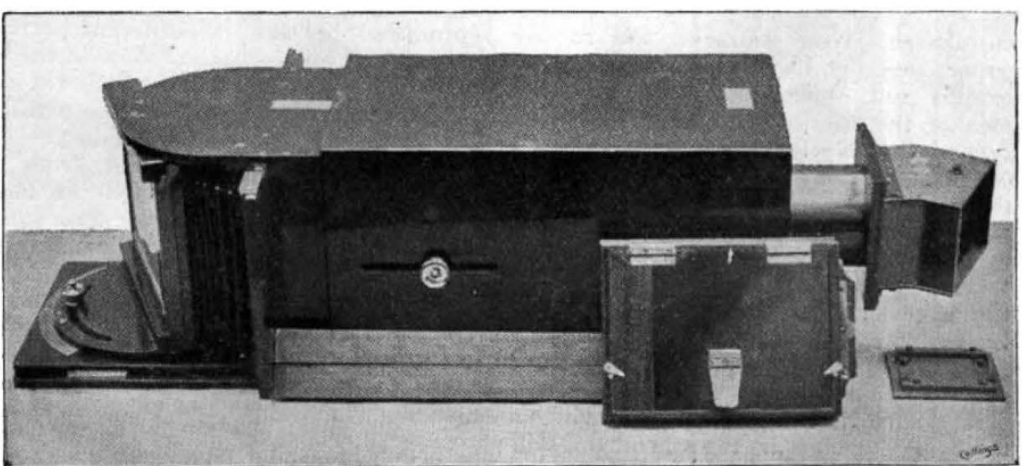

Fig. 7.-Quartz-Calcite Prismatic Camera.

Conclusions as to the temperatures of the stars, as was suggested by Crova in 1878,4 may therefore be based on I Roy. Soc. Proc., vol. Ixxiii. p. 227 (rgo4).

2 Roy. Soc. Proc., vol. xxiv. p. 353 (1876).

3 It has been observed that various metals, including iron, produce a continuous absorption at the blue end of the spectrum when reduced to the state of vapour in the relatively cool oxy-bydrogen flame (Lockyer and Roberts-Austen, Roy. Soc. Proc, vel. xxiii. p. 344, 1875).

${ }_{4}$ Comptes rendus, vol, xvii. p. $98 \mathrm{r}$. 
the relative intensities of different parts of the continuous spectrum. While this was in reality what had already been done in the case of spectra photographed with glass prisms, the new apparatus permitted comparisons over a much longer range. In each case the limits of the spectra towards the ultra-violet in the photographs are determined by the intensities of the spectra in that region and the duration of the exposures.

To eliminate as far as possible the varying effects of atmospheric absorption, to which the ultra-violet rays are specially sensitive, and the errors which might arise from differences in photographic treatment, each selected pair of stars was photographed on the same plate when the stars had approximately the same altitude, and if any change in the atmospheric conditions were suspected the result was discarded. In each case an attempt was made so to expose the photographs that in every pair the intensity of the spectrum was as nearly as possible the same in both stars in the region between $\mathrm{H} \beta$ and $\mathrm{H} \gamma$. This condition was very difficult to fulfil in actual practice, owing to the different magnitudes and declinations of the stars compared, and the consequent need for very careful adjustment of the clock rate. The difficulty was further increased on account of the different actinism of the stars in this part of the spectrum. The work, however, has resulted in a series of comparison photographs from which all variable conditions except the natural variations in radiation have, so far as possible, been less extended towards the ultra-violet than that of $\eta$ Urse Majoris, and the maximum intensity is much nearer the red end.

The general result of this research is thus stated:"Taking the stars assumed to be hottest in the chemical classification, we find that in all cases the relative length of the spectrum is reduced, and the relative intensity of the red is increased, as a lower temperature is reached. That is to say that where two spectra having their intensities about the region $\mathrm{H} \beta-\mathrm{H} \gamma$ equal are compared, we find that in the cooler stars, according to the chemical classification, the emissions in the red preponderate, whilst in the hotter star the ultra-violet is more extended and intense.'

In other words, the sequence of the various groups of stars, as determined by this more extended study of the continuous radiations, so far as the investigation has yet been carried, is identical with that previously arrived at from a discussion of the line spectra. It follows, therefore, that the classification which was based upon the chemical differences indicated by the successive appearances of metallic, protometallic, and gaseous lines, in all probability reveals also the true temperature sequence of the different varieties of stars.

As pointed out by Sir Norman Lockyer, this result is at variance with that arrived at by Sir William and Lady Huggins. While also basing their conclusions as to relative stellar temperatures on the comparative intensities
Ultra-violet.

Violet.
Blue.

Red.
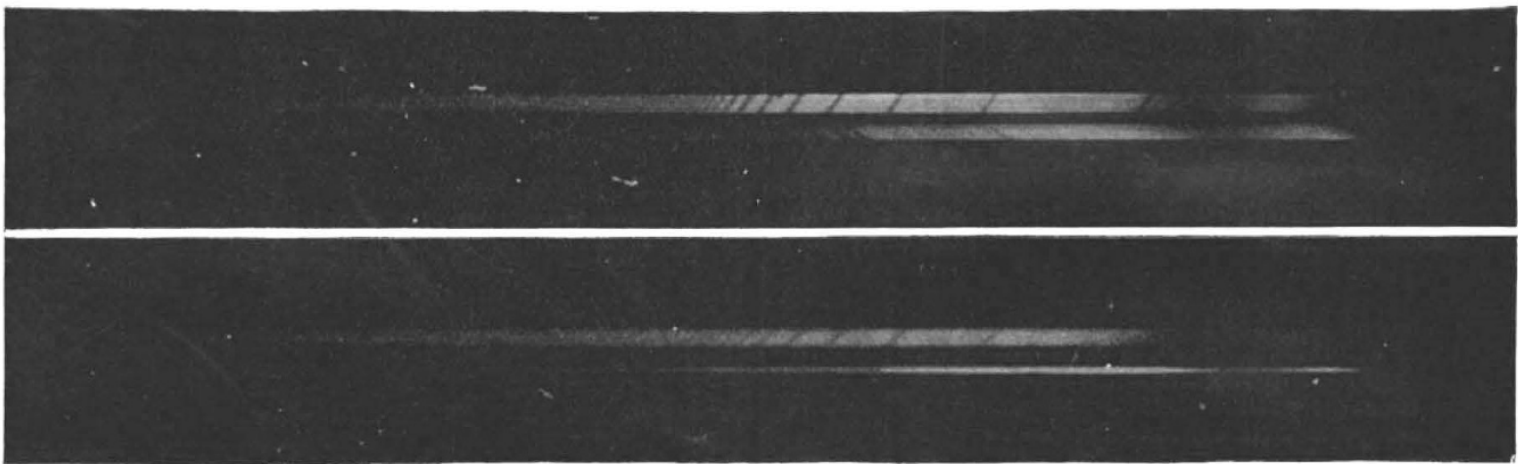

FIG. 8.-Comparison of the spectra of pairs of stars at different temperature stages. Enlarged $3 \frac{1}{2}$ times from the original negatives taken at the Solar Phy ics Observatory, South Kensington. (1) Vega, stage 4 ; Arcturus, stage 2. (2) $\eta$ Ursæ Majoris, stage 8 ; Capeila, stage 2

eliminated. Nine pairs of spectra are reproduced in the paper, and detailed descriptions of these, and other photographs not reproduced, are given. A sufficiently clear idea of the results may be gathered from Fig. 8, showing two of the selected pairs of stars. It is necessary to point out that as the plates employed were but little sensitive to the green rays, there is a break in each spectrum from about $\lambda 486$ to $\lambda 550$, followed on the less refrangible side by a further portion of the spectrum having its centre about "D." The numbered "stages" in the description refer to the ten horizons of mean temperature already shown in Fig. 6, stage I corresponding to the fluted spectra of the Antarian and Piscian stars, and stage to to the simplified spectra of the $\gamma$ Argus type.

A glance at the photographs will suffice to show that in the case of each pair the star at the higher stage of temperature, as previously determined from the investigation of the line spectra, has the greater development of the violet end of the spectrum, and that the difference is more marked the greater the temperature difference. In the first example it will be seen that, while the maximum intensity of the spectrum of Vega is in the blue, that of Arcturus is obviously much further towards the red end; the differences at the extremities of the spectrum are also very marked, Vega having the greater extension into the ultra-violet, and a relatively reduced intensity at the red end as compared with Arcturus. Again, in the second pair, in spite of relative over exposure, the spectrum of Capella is notably NO. I 826 , VOL. 70] of different parts of the continuous spectra, and recognising that some of the stars must be getting hotter, these observers have concluded that the highest temperature is to be found not in the white stars, but in stars resembling the sun. They write ${ }^{1}$ :- " If the relative intensity of this part of the spectrum, from about $K$ onwards to about $\lambda 3400$, be regarded as an indication of temperature, we should have to consider Procyon as at a hotter stage than Vega, and that the highest stage of temperature is reached in the true solar stage, of which Capella is typical. Then a fall of temperature sets in, as is shown in the advancing enfeeblement of this part of the spectrum in Arcturus, Betelgeuse, and Aldebaran." Special stress is laid by these observers on "the rather sudden fall of intensity of the continuous spectrum at about the place of the end of the series of dark hydrogen lines" in such stars as Vega (a feature which is well brought out in the photograph of the spectrum of this star reproduced in Fig. 8), but Sir Norman Lockyer states that this in no way affects his results, and promises another paper dealing with this and similar points. The precautions taken by Sir Norman Lockyer to secure equal treatment for the stars compared would seem to give his results greater weight than those of the other observers, whose photographs appear to have been obtained in the course of more or less routine work on the spectra of individual stars.

It should also be noted that the occurrence of protometallic lines has not been accepted as evidence of the 1 "Atlas of Representative Stellar Spectra," p. 85 . 
relatively high temperature of a star by some observers, in consequence of the production of these lines in arc spectra under certain special conditions. The recent work of $\mathrm{Mr}$. C. de Watteville ${ }^{2}$ on flame spectra in relation to spark spectra obtained with and without self-induction, however, appears to be entirely in harmony with the result derived from stellar inquiries by Sir Norman Lockyer.

At present, then, the evidence available appears to favour the view that the chemical differences indicated in the different groups of stars are due to differences of temperature, and that successively higher stages of heat are indicated by the predominance of metallic, proto-metallic, and gaseous lines. Thus, although further researches on some points are needed, it is very probable that the new classification correctly exhibits the relative temperatures of the various stellar groups, besides giving exhaustive definitions and providing a convenient nomenclature. At the same time, the sequence of phenomena indicated in the classification seems strongly to support the dissociation hypothesis.

A. FOWLER.

\section{THE FALKLAND ISLANDS} AND THEIR FAUNA.

MR. RUPERT VALLENTIN, who has spent many years in the Falklands, where he has been an assiduous observer and collector of the fauna and flora, contributes an excellent account, illustrated by photographs, of those remote islands to the third part of vol. xlviii. of the Memoirs of the Manchester Literary and Philosophical Society, of which his article forms No. 23. The author alludes in the first place to the celebrated stone-rivers, which consist of slowly moving blocks of quartzite between banks of peat. In Mr. Vallentin's opinion the stones forming these "rivers" had approximately attained their present position before the formation of the peat, and the "rivers"

ing, the sole remnant of the covering of vegetation is a mass of bog-balsam (Bolax globaria), as shown in Fig. $x$, which, owing to its long tapering root, can obtain nourishment after the soil which supported other plants has been washed away. Very similar "stone-rivers" exist in parts of the Himalaya.

Apparently the Falklands are by no means the desolate

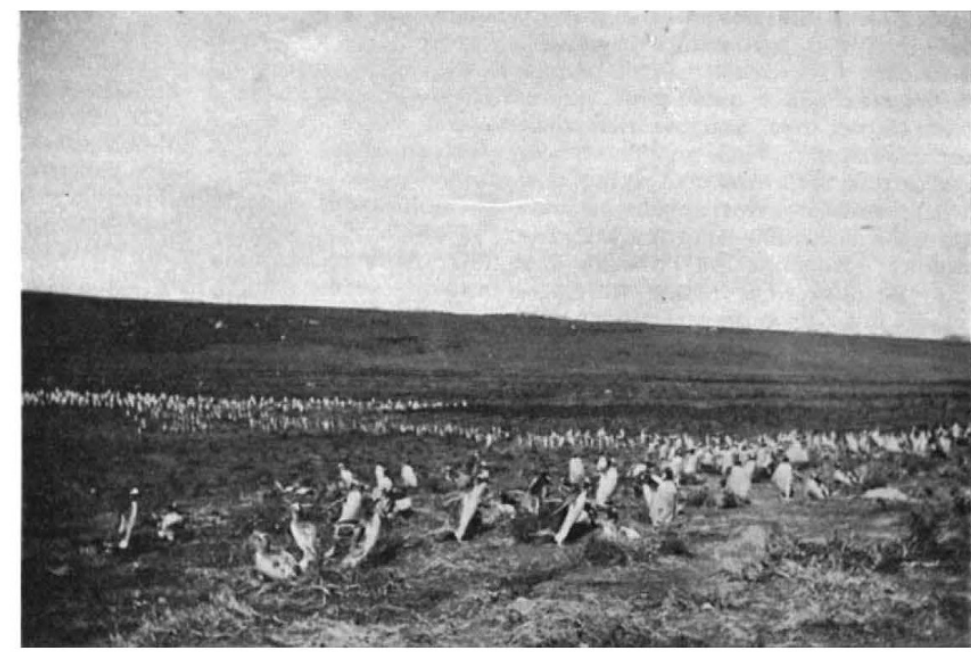

Fig, 2.--Gentu Penguins on the Falklands. The birds in the background are running to the shore.

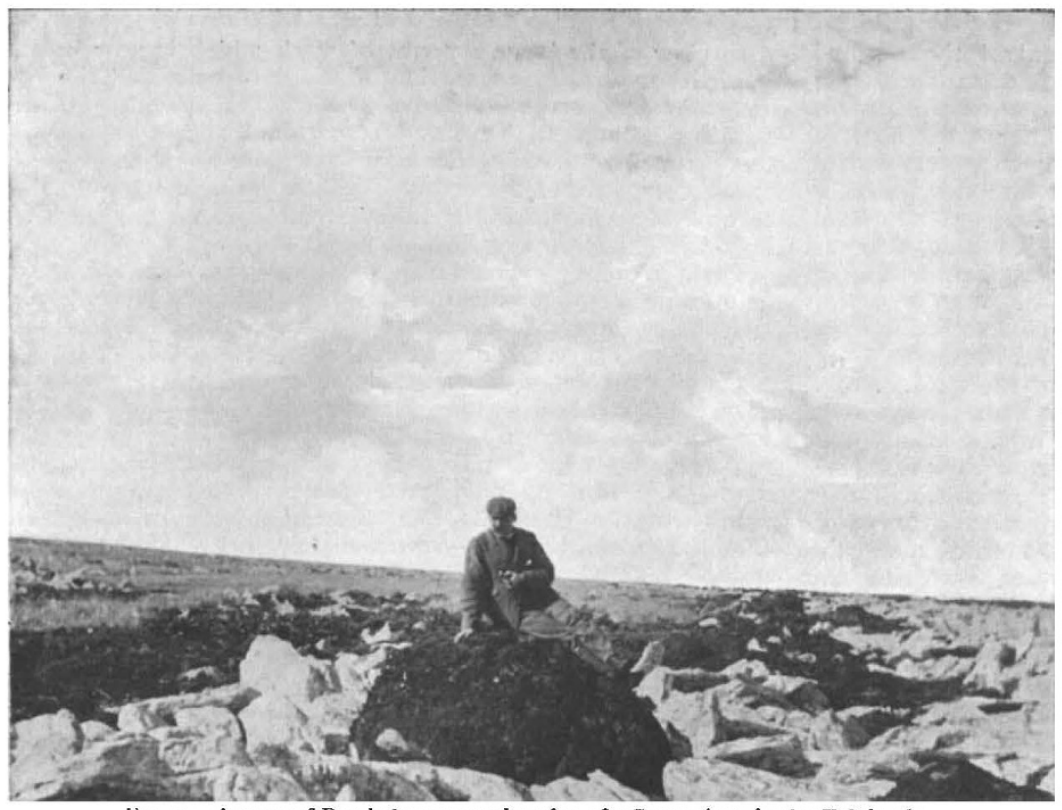

Fig. I.-A mass of Bog-balsam near the edge of a Stone-river in the Falklands.

spots we are often prone to imagine, the vegetation being locally abundant, and the shores of the estuaries and coves on part of West Falkland being fringed with bushes of the attractive Falkland box (Veronica decurrata), which has a beautiful and highly scented flower. With the aid of abundant manure, many English vegetables can be grown in sheltered spots.

With regard to the fauna, the most interesting statement is the one to the effect that, so far as the author could ascertain, the Falkland Island wolf (Canis antarcticus) is now completely exterminated. This latest addition to the list of animals extirpated in recent years by human agency is the more to be regretted seeing that this wolf, or fox as it used to be called by the settlers, is an extremely interesting animal from the point of view of geographical distribution, and one that is probably very insufficiently represented in our museums. According to Prof. Huxley's paper on the dentition of the Canidæ, published in the Zoological Society's Proceedings for I880, the Falkland Island wolf is closely allied to the North American coyote, the remarkable feature connected with this resemblance being that there are no true wolves in either Central or South America. The British Museum has one mounted skin of the Antarctic wolf in the exhibition galleries, and there are two skeletons in the store collection. Strychnine poisoning appears to have brought about the extermination of this wolf, the last survivor have been produced by the denudation of the peat. In of which seems to have been killed so long ago as 1876 . every "stone-river" islets of vegetation remain near the margins, these being most luxurious where the denudation has been recent. Where the denudation is of long stand-

1 Roy. Soc. Proc., vol. lxxiv. p. 85 (rgo4).

Birds form by far the most important portion of the terrestrial vertebrate fauna of the Falklands, and among these penguins, of three species, and "mollymauks," or lesser albatrosses, are numerically the most abundant. Mr.

No. I 826 , VOL. 70$]$ 